\title{
The Darboux transformation and algebraic deformations of shape-invariant potentials.
}

\author{
D Gómez-Ullate†, N Kamran $\ddagger$ and R Milson $\S$ \\ † Centre de Recherches Mathématiques, Université de Montréal, (QC) H3C 3J7 \\ Canada. \\ $\ddagger$ Department of Mathematics and Statistics, McGill University, Montréal (QC) H3A \\ 2K6 Canada. \\ $\S$ Department of Mathematics and Statistics, Dalhousie University, Halifax (NS) B3H \\ 3J5 Canada. \\ E-mail: ullate@crm.umontreal.ca, nkamran@math.mcgill.ca, \\ milson@mathstat.dal.ca
}

\begin{abstract}
We investigate the backward Darboux transformations (addition of a lowest bound state) of shape-invariant potentials on the line, and classify the subclass of algebraic deformations, those for which the potential and the bound states are simple elementary functions. A countable family, $m=0,1,2, \ldots$, of deformations exists for each family of shape-invariant potentials. We prove that the $m^{\text {th }}$ deformation is exactly solvable by polynomials, meaning that it leaves invariant an infinite flag of polynomial modules $\mathcal{P}_{m}^{(m)} \subset \mathcal{P}_{m+1}^{(m)} \subset \ldots$, where $\mathcal{P}_{n}^{(m)}$ is a codimension $m$ subspace of $\left\langle 1, z, \ldots, z^{n}\right\rangle$. In particular, we prove that the first $(m=1)$ algebraic deformation of the shape-invariant class is precisely the class of operators preserving the infinite flag of exceptional monomial modules $\mathcal{P}_{n}^{(1)}=\left\langle 1, z^{2}, \ldots, z^{n}\right\rangle$. By construction, these algebraically deformed Hamiltonians do not have an $\mathfrak{s l}(2)$ hidden symmetry algebra structure.
\end{abstract}

PACS numbers: 03.65.Fd, 03.65.Ge 


\section{Introduction.}

The Darboux transformation [1, p. 210] [2], a method based on the factorization of second-order operators, is an important technique for the exact solution of the onedimensional Schrödinger equation [3, 4]. The transformation is also a key concept in supersymmetric quantum mechanics [5] and the theory of integrable systems [6, 7, 8]. From the point of view of spectral theory, a non-singular Darboux transformation can be characterized by the following three possibilities 9]. First, a potential with a lowest bound state admits a unique forward Darboux transformation, which deletes the ground state. Second, a potential admits a 2-parameter family (the energy and a shape parameter) of backward Darboux transformations, each of which adds a lowest eigenvalue. Third, there is also a 1-parameter family of isospectral transformations, corresponding to two critical values of the shape parameter, which neither add nor delete bound states [10].

The Darboux transformation relates to exact solutions in several ways [11]. Generally, the forward transformation deforms a given exactly solvable potential to a new, solvable potential form. However, if a parameterized family of exactly solvable potentials is shape-invariant [12], i.e., closed with respect to the forward Darboux transformation, then the forward transformation furnishes an explicit description of the spectrum and eigenfunctions, rather than a new exactly-solvable form. Therefore, to obtain a solvable deformation of a shape-invariant potential it is necessary to employ a backward Darboux transformation. This was first done for specific potentials such as the harmonic oscillator [13], while the general theory was developed in [6, 9].

However, the general form of the deformed potential features integrals of eigenfunctions of the original Hamiltonian - in contrast to the original potential, which is an elementary function, with bound states also described by elementary functions. It has been noted [14] that only certain discrete values of the energy and shape parameter correspond to an algebraic deformation, one where the potential and the bound states remain elementary functions. Such forms are attractive from the modeling standpoint, and are also important theoretically, since exact results can be obtained even in critical conditions, where numerical techniques break down.

In the present article we explain the discrete nature of algebraic deformations by characterizing such deformations in terms of polynomial modules left invariant by a second-order differential operator. The invariant module approach is an alternative, inherently algebraic, approach to exact solvability — developed originally to treat quasiexactly solvable Hamiltonians [15, 16, 17]. In this approach, one considers a Hamiltonian that, in a suitable gauge, preserves an infinite flag of polynomial modules

$$
\mathcal{P}_{0} \subset \mathcal{P}_{1} \subset \mathcal{P}_{2} \subset \ldots \subset \mathcal{P}_{n} \subset \ldots, \quad \mathcal{P}_{n}=\left\langle 1, z, \ldots, z^{n}\right\rangle .
$$

Such a Hamiltonian has an upper-triangular action, and it is, therefore, algebraically diagonalizable. On the line, there are exactly three potential forms whose Hamiltonian, in a suitable gauge, preserves (1). These are the classical shape-invariant potential families: the harmonic oscillator, the Morse [18, and the hyperbolic Pöschl-Teller [19] 
potentials. This invariant module approach provides an alternative explanation for the exact-solvability of these shape-invariant potentials.

The details of this approach can be found in [15, 17], and some generalizations in [20]. The method of invariant polynomial flags has also been applied in quantum many-body problems 21. The question of determining which second-order operators preserve a finite-dimensional polynomial module has been previously considered in a number of papers, including [22,23].

We consider the algebraic deformations of the three shape-invariant potential families [11,14,24, and show that, in each case, the $m^{\text {th }}$ algebraic deformation produces a Hamiltonian that, modulo a gauge transformation and a change of variable, preserves an infinite flag of deformed polynomial modules

$$
\mathcal{P}_{m}^{(m)} \subset \mathcal{P}_{m+1}^{(m)} \subset \mathcal{P}_{m+2}^{(m)} \subset \ldots \subset \mathcal{P}_{n}^{(m)} \subset \ldots
$$

where each $\mathcal{P}_{n}^{(m)}$ is a certain codimension $m$ subspace of $\mathcal{P}_{n}$, i.e. the span of $n-m+1$ polynomials of degree $n$.

We study the first deformation $(m=1)$ in some detail, and show that $\mathcal{P}_{n}^{(1)}$ is an exceptional monomial module [22], an invariant vector space spanned by

$$
1, z^{2}, z^{3}, \ldots, z^{n} \quad \text { (the first power is omitted). }
$$

We also show that higher deformations produce more complicated polynomial modules, but we do not analyze these modules here.

We will discuss monomial modules more thoroughly in a forthcoming publication 25. At this point, we would like to mention that exceptional monomial modules also arise in the context of N-fold supersymmetry, 26]. Our emphasis is somewhat different, since we are primarily concerned with the interplay between the backward Darboux transformation and the class of the operators preserving an infinite flag of polynomial modules.

We also note that invariance of (11) is generally achieved by expressing the gauge hamiltonian as a quadratic combination of those generators of $\mathfrak{s l}(2)$, realized as first order differential operators, which leave invariant the infinite flag of polynomial subspaces $\mathcal{P}_{n}$. These operators are called Lie-algebraic and the Hamiltonian is said to have a hidden $\mathfrak{s l}(2)$ symmetry algebra. Lie-algebraic potentials in one dimension have been classified in [17. However, not all exactly-solvable potentials are generated by a hidden symmetry, the Coulomb potential being a notable counterexample [27]. Since the potentials studied here preserve (2) rather than (11), they lack an $\mathfrak{s l}(2)$ hidden symmetry algebra structure, and thus furnish a further indication that the exactly solvable class of potentials is larger than the Lie algebraic one [15, 16, 17.

This paper is structured as follows. In the next Section we describe the forward and backward Darboux transform. In Section 3 we discuss exactly solvable operators and algebraic deformations. We also exhibit the invariant flags corresponding to the algebraic deformations of the three shape-invariant potential families. In Section 4 we consider exactly solvable operators that preserve the exceptional monomial module, and 
demonstrate that these are precisely the first-fold deformations of the shape-invariant Hamiltonians discussed in Section 3 .

\section{Factorization and the Darboux transformation}

Let $U(x), x \in(-\infty, \infty)$ be a continuous, real-valued function, and let

$$
\tau=-\partial_{x x}+U
$$

be the corresponding formally self-adjoint differential operator. We fix a formal eigenfunction

$$
\tau[\phi]=\lambda_{0} \phi
$$

and note that every such $\phi$ corresponds to a differential factorization

$$
\tau-\lambda_{0}=\alpha^{*} \alpha
$$

where

$$
\alpha=\partial_{x}+\sigma_{x}, \quad \alpha^{*}=-\partial_{x}+\sigma_{x}, \quad \sigma=-\ln \phi .
$$

For this reason we shall refer to $\phi$ as the factorization function, and to $\lambda_{0}$ as the factorization energy.

Commutation of the factors defines a partner differential operator

$$
\hat{\tau}=\alpha \alpha^{*}=-\partial_{x x}+\hat{U}
$$

where

$$
\hat{U}=U+2 \sigma_{x x}
$$

The operators obey the following intertwining relation:

$$
\alpha \tau=\hat{\tau} \alpha .
$$

As a consequence, the first-order operator $\alpha$ relates the eigenfunctions of the two operators: given

$$
\tau[\psi]=\lambda \psi
$$

we also have

$$
\hat{\tau}[\hat{\psi}]=\lambda \hat{\psi}, \quad \hat{\psi}=\alpha[\psi] .
$$

To give a rigorous treatment of the Darboux transformation [7, 28, we assume that $U(x)$ is bounded from below, and let $\mathcal{H}$ be the unique self-adjoint, semi-bounded

operator corresponding to $\tau$. The partner potential $\hat{U}(x)$ is continuous if and only if $\phi$ is non-vanishing. In this case, the spectrum of $\hat{U}(x)$ is bounded from below, and we let $\hat{\mathcal{H}}$ denote the corresponding self-adjoint, semi-bounded operator. Letting $A$ denote the closed operator corresponding to $\alpha$, we have that $\alpha^{*}$ corresponds to the adjoint $A^{*}$. We therefore obtain the following non-formal factorizations:

$$
\mathcal{H}-\lambda=A^{*} A, \quad \hat{\mathcal{H}}-\lambda=A A^{*},
$$


where the compositions are appropriately restricted.

In particular, $A$ maps $D(\mathcal{H})$, the domain of $\mathcal{H}$, to $D(\hat{\mathcal{H}})$. The spectral properties of this transformation are governed by one of the following 3 possibilities [6].

(i) Forward transformation: $\phi$ is square integrable, and $A$ defines an isomorphism between ker $A=\phi^{\perp}$ and $D(\hat{\mathcal{H}})$. Thus, a forward transformation exists if and only if $\mathcal{H}$ possesses a discrete spectrum, in which case it is unique. The transformed spectrum differs from the spectrum of $\mathcal{H}$ by the removal of $\lambda$, the lowest eigenvalue. The first-order operator $\alpha$ transforms the $n^{\text {th }}$ bound state of $\mathcal{H}$ to the $(n-1)^{\text {st }}$ bound state of $\hat{\mathcal{H}}$.

(ii) Backward transformation: $\phi^{-1}$ is square integrable, and $A$ defines an embedding of $D(\mathcal{H})$ into $D(\hat{\mathcal{H}})$. The range of the embedding is precisely $\left(\phi^{-1}\right)^{\perp}$. The spectrum of $\hat{\mathcal{H}}$ differs from that of $\mathcal{H}$ by the addition of a lowest eigenvalue, namely $\lambda$, with the ground state given by $\phi^{-1}$. The operator $\alpha$ transforms the $n^{\text {th }}$ bound state of $\mathcal{H}$ to the $(n+1)^{\text {st }}$ bound state of $\hat{\mathcal{H}}$.

A 1-parameter family of backward transformations exist for every $\lambda$ strictly smaller than the spectrum of $\mathcal{H}$. To describe the possibilities, let $\phi_{+}$and $\phi_{-}$be the unique (up to a multiple) positive solutions of

$$
\tau\left[\phi_{ \pm}\right]=\lambda \phi_{ \pm}
$$

with the property that $\phi_{ \pm}$is square integrable near, respectively, $\pm \infty$. The desired $\phi$ is of the form

$$
\phi=s \phi_{+}+t \phi_{-}, \quad s, t>0 .
$$

(iii) Isospectral transformation: neither $\phi$ nor $\phi^{-1}$ are square integrable. In this case $A$ acts as an isomorphism between $D(\mathcal{H})$ and $D(\hat{\mathcal{H}})$. The operator $\alpha$ transforms the $n^{\text {th }}$ bound state of $\mathcal{H}$ to the $n^{\text {th }}$ bound state of $\hat{\mathcal{H}}$. Two isospectral Darboux transformations exist for every $\lambda$ strictly smaller than the spectrum of $\mathcal{H}$ : one corresponding to $\phi=\phi_{+}$, and the other to $\phi=\phi_{-}$.

\section{Algebraic deformations of shape-invariant potentials.}

\subsection{Exact solvability}

We will call a Schrödinger operator

$$
\mathcal{H}=-\partial_{x x}+U
$$

exactly solvable by polynomials if $\mathcal{H}$ is equivalent, by a change of variable and a gauge transformation, to a second-order operator $T$ that preserves an infinite flag of finitedimensional polynomial modules

$$
\mathcal{M}_{1} \subset \mathcal{M}_{2} \subset \mathcal{M}_{3} \subset \ldots, \quad \operatorname{dim} \mathcal{M}_{n}=n .
$$


The exact solvability comes about because $T$ is upper-triangular relative to a basis adapted to the above flag, and hence possesses an infinite list of eigenpolynomials. In this paper we will make the hypothesis that such an operator is of the form

$$
T=P(z) \partial_{z z}+Q(z) \partial_{z z}+R(z)
$$

where

$$
P(z)=p_{2} z^{2}+p_{1} z+p_{0}, \quad p_{0}, p_{1}, p_{2} \in \mathbb{R},
$$

is a polynomial of degree 2 or less, and where $Q(z), R(z)$ are rational functions.

We transform the second-order eigenvalue equation

$$
T[f]=\lambda f
$$

into a Schrödinger equation

$$
\mathcal{H}[\phi]=\lambda \phi
$$

by a change of variables

$$
x=\int^{z}(-P)^{-\frac{1}{2}}
$$

and a gauge transformation

$$
\phi=\left.e^{p} f\right|_{z=z(x)}
$$

where

$$
\begin{aligned}
& p=\int^{z} \frac{1}{2} P^{-1}\left(Q-\frac{1}{2} P^{\prime}\right) \\
& U=\frac{1}{4} P^{\prime \prime}-\frac{1}{2} Q^{\prime}-\frac{1}{4} P^{-1}\left(Q-\frac{1}{2} P^{\prime}\right)\left(Q-\frac{3}{2} P^{\prime}\right)+\left.R\right|_{z=z(x)}
\end{aligned}
$$

Let $\mathcal{R}$ denote the range of the function $z(x)$, and let us assume that neither $Q(z)$ nor $R(z)$ have singularities for $z \in \mathcal{R}$. We assume that $P(z)$ is not everywhere positive. If it were, then we change $P \rightarrow-P$. With these assumptions we prove the following

Proposition 1 Exactly one of the following possibilities holds, according to the number and multiplicity of the real roots of $P(z)$ :

(i) The are no real roots. Then, $\mathcal{R}=(-\infty, \infty)$, and $U(x)$ is non-singular.

(ii) There is a double root, $\rho$. Then, $\mathcal{R}=(\rho, \infty)$ or $(-\infty, \rho)$, and $U(x)$ is non-singular.

(iii) There is a unique, simple root, $\rho$. Then, $\mathcal{R}=(-\infty, \rho]$, or $[\rho, \infty)$. Both $z(x)$ and $U(x)$ are even functions. The potential is non-singular if and only if

$$
Q(\rho)=\frac{1}{2} P^{\prime}(\rho), \quad \text { or } \quad Q(\rho)=\frac{3}{2} P^{\prime}(\rho)
$$

(iv) There are two distinct roots $\rho_{1}<\rho_{2}$, and $\mathcal{R}=\left(-\infty, \rho_{1}\right]$, or $\left[\rho_{2}, \infty\right)$. Then, both $z(x)$ and the potential are even functions. The potential is non-singular if and only if (12) holds with, $\rho=\rho_{1}$, or with $\rho=\rho_{2}$, respectively.

(v) There are two distinct roots $\rho_{1}<\rho_{2}$, and $\mathcal{R}=\left[\rho_{1}, \rho_{2}\right]$. Then, both $z(x)$ and the potential are periodic functions, and $U(x)$ has a singularity. 
Proof. Condition (12) follows from (11). In order for the potential to be nonsingular, the function $\left(Q-\frac{1}{2} P^{\prime}\right)\left(Q-\frac{3}{2} P^{\prime}\right)$ must vanish at those roots of $P(z)$ that lie in $\mathcal{R}$. The singularity occurs in case $(v)$, because both $Q(z)$ and $P^{\prime}(z)$ are first-degree polynomials, and hence (12) cannot hold for both $\rho=\rho_{1}$ and $\rho=\rho_{2}$.

\subsection{Shape-invariant potentials on the line.}

Let us begin by showing, that the class of exactly solvable Hamiltonians that preserve the infinite flag (11) consists of shape-invariant potentials on the line. Up to a constant, the most general form of a second-order operator that preserves (11) is

$$
T=P(z) \partial_{z z}+Q(z) \partial_{z}
$$

where $P(z)$ and $Q(z)$ are arbitrary second and first degree polynomials, respectively. Since $\mathcal{P}_{n}$ is invariant with respect to affine transformations of the $z$ variable, we can use such transformations to modify $P(z)$ and $Q(z)$ without loss of generality. Additionally, by rescaling the $x$ variable, and shifting the spectrum, we reduce our analysis to one of the following four canonical forms:

\begin{tabular}{c|cccc} 
& $P(z)$ & $Q(z)$ & $z(x)$ & $U(x)$ \\
\hline Ia & -1 & $2 z$ & $x$ & $x^{2}$ \\
Ib & $-4 z$ & $4 z-2$ & $x^{2}$ & $x^{2}$, \\
II & $-z^{2}$ & $-(2 A+3) z+1$ & $e^{x}$ & $\frac{1}{4} e^{-2 x}-\left(A+\frac{1}{2}\right) e^{-x}$, \\
III & $z(1-z)$ & $\left(A-\frac{3}{2}\right) z+1-A$ & $\cosh ^{2}\left(\frac{x}{2}\right)$ & $\frac{1}{4}\left(\frac{1}{4}-A^{2}\right) \operatorname{sech}^{2}\left(\frac{x}{2}\right)$.
\end{tabular}

Table 1. Shape-invariant potentials on the line

By Proposition 1, the $Q(z)$ in cases Ia, Ib, and III is the most general form for which $U(x)$ is non-singular. In case II, we use a translation of the $x$ variable to fix the form of $Q(z)$. Thus, we have derived the classical shape-invariant potential families: the harmonic oscillator (Ia) (Ib), the Morse potential (II), and the hyperbolic Pöschl-Teller potentials (III). Each of these potential forms is discussed in more detail below.

Let us now show that these potentials are shape-invariant by construction. A parameterized family of potentials is called shape-invariant if the ground state Darboux transformation acts by changing the potential parameters, but leaves the form of the potential invariant. For the operators in question, the ground state energy is $\lambda=0$, and hence the corresponding factorization is given by

$$
\mathcal{H}=\left.\left(-(-P)^{\frac{1}{2}} \partial_{z}+\frac{1}{2}\left(Q-\frac{1}{2} P^{\prime}\right)(-P)^{-\frac{1}{2}}\right)\left((-P)^{\frac{1}{2}} \partial_{z}+\frac{1}{2}\left(Q-\frac{1}{2} P^{\prime}\right)(-P)^{-\frac{1}{2}}\right)\right|_{z=z(x)},
$$

where $z=z(x)$ is the change of variable defined in (8) . The commutation of the factors produces a Schrödinger operator $\hat{\mathcal{H}}$, which corresponds to the algebraic operator

$$
\hat{T}=P(z) \partial_{z z}+\hat{Q}(z) \partial_{z}
$$

where

$$
\hat{Q}=P^{\prime}-Q
$$


For this reason, the forward Darboux transformation for these potentials produces another potential (11) of the same form, but with potential parameters modified by (14).

\subsection{Algebraic deformations.}

Now, let us isolate the values of the energy and shape parameters for which the backward Darboux transformation of the shape-invariant potentials yields an algebraic deformation. We will say that a Darboux transformation is an algebraic deformation, when the derivative of $\ln \phi$ in (3) is either a rational function, or a composition of a rational function with an exponential. As per (99)(13), for the shape-invariant potentials under discussion, the factorization function is of the form

$$
\phi(x)=e^{p(z(x))} f(z(x)),
$$

where $p(z)$ is a polynomial, $z(x)$ is an elementary function, and $f(z)$ is a hypergeometric function. We will say that such an $f(z)$ is of polynomial type if $f^{\prime}(z) / f(z)$ is a rational function. We observe that

$$
(\ln \phi(x))^{\prime}=p^{\prime}(z(x)) z^{\prime}(x)+\frac{f^{\prime}(z(x))}{f(z(x))} z^{\prime}(x) .
$$

Thus, to obtain an algebraic deformation we must demand that $f(z)$ be of polynomial type, with $f(z)$ non-vanishing in the range $\mathcal{R}$ of $z(x)$ (see Proposition 11).

The regular solution of the confluent hypergeometric equation [29, Sec. 6.1],

$$
z y^{\prime \prime}(z)+(c-z) y^{\prime}(z)-a y(z)=0,
$$

is given by the confluent hypergeometric function

$$
y(z)=\Phi(a, c, z)={ }_{1} F_{1}(a, c ; z) .
$$

It can be shown 29, Sec. 6.9] that solutions of polynomial type exist if and only if either

$a$ or $c-a$ is an integer. These solutions, expressed in terms of generalized Laguerre polynomials $L_{m}^{a}(z), m=0,1,2, \ldots$, are given below:

$$
\begin{aligned}
y_{1}(z) & =\Phi(a, c ; z) & & a=-m, \\
& \propto L_{m}^{c-1}(z) & & \\
y_{2}(z) & =z^{1-c} \Phi(a-c+1,2-c ; z) & & c-a=1+m, \\
& \propto z^{1-c} L_{m}^{1-c}(z) & & c-a=-m, \\
y_{3}(z) & =e^{z} \Phi(c-a, c ;-z) & & \\
& \propto e^{z} L_{m}^{c-1}(-z) & & a=m, \\
y_{4}(z) & =z^{1-c} e^{z} \Phi(1-a, 2-c,-z) & & \propto z^{1-c} e^{z} L_{m}^{1-c}(-z)
\end{aligned}
$$

In general, we have $y_{1}(z)=y_{3}(z)$ and $y_{2}(z)=y_{4}(z)$ [29, Sec. 6.4].

The regular solution of the hypergeometric equation [29, Sec. 6.1],

$$
z(1-z) f^{\prime \prime}(z)+(c-(a+b+1) z) f^{\prime}(z)-a b f(z)=0,
$$


is given by the Gauss hypergeometric function

$$
f(z)=F(a, b, c ; z)={ }_{2} F_{1}(a, b, c ; z) .
$$

Solutions of polynomial type exist if and only if (17) is of so-called degenerate type. This means that the monodromy group around one of the regular singular points $0,1, \infty$ is trivial [29, Sec. 2.2]. These solution, expressed in terms of Jacobi polynomials $P_{m}^{(\alpha, \beta)}(z)$, are shown below:

$$
\begin{aligned}
f_{1}(z) & =F(a, b, 1+a+b-c ; 1-z), & & a=-m, \\
& \propto P_{m}^{(\alpha, \beta)}(2 z-1) & & \alpha=b-c-m, \beta=c-1 \\
f_{2}(z) & =z^{1-c}(1-z)^{c-a-b} F(1-a, 1-b, 1-a-b+c ; 1-z) & & a=m+1, \\
& \propto z^{\beta}(1-z)^{\alpha} P_{m}^{(\alpha, \beta)}(2 z-1) & & \alpha=c-b-1-m, \beta=1-c \\
f_{3}(z) & =(1-z)^{c-a-b} F(c-a, c-b, 1-a-b+c ; 1-z) & & c-a=-m, \\
& \propto(1-z)^{\alpha} P_{m}^{(\alpha, \beta)}(2 z-1), & & \alpha=-b-m, \beta=c-1 \\
f_{4}(z) & =z^{1-c} F(a+1-c, b+1-c, 1+a+b-c ; 1-z) & & c-a=m+1, \\
& \propto z^{\beta} P_{m}^{(\alpha, \beta)}(2 z-1), & & \alpha=b-1-m, \beta=1-c
\end{aligned}
$$

We will need (16) to construct algebraic deformations of the Harmonic oscillator and the Morse potential, and (18) to construct the algebraic deformations of the hyperbolic Pöschl-Teller potential.

\subsection{The Harmonic oscillator.}

The general solution of

$$
-\phi^{\prime \prime}(x)+U_{\mathrm{ho}}(x) \phi(x)=\lambda \phi(x), \quad U_{\mathrm{ho}}(x)=x^{2},
$$

is given by

$$
\phi_{\text {ho }}\left(x ; \lambda, A_{0}, A_{1}\right)=\left(A_{0} \Phi\left(\frac{1}{4}-\frac{\lambda}{4}, \frac{1}{2} ; x^{2}\right)+A_{1} x \Phi\left(\frac{3}{4}-\frac{\lambda}{4}, \frac{3}{2}, x^{2}\right)\right) \mathrm{e}^{-\frac{x^{2}}{2}},
$$

The $n^{\text {th }}$ bound state is

$$
\psi_{\mathrm{ho}, n}(x) \propto e^{-\frac{x^{2}}{2}} H_{n}(x) \propto \begin{cases}\phi_{\mathrm{ho}}(x ; 1+2 n, 1,0), & n \text { even }, \\ \phi_{\mathrm{ho}}(x ; 1+2 n, 0,1), & n \text { odd },\end{cases}
$$

where $H_{n}(z)$ denotes the $n^{\text {th }}$ Hermite polynomial. For $\lambda<1$, the nodeless solutions of (19) are given by

$$
\phi_{\text {ho }}(x ; \lambda, 1, t), \quad|t| \leq 2 \frac{\Gamma\left(\frac{3}{4}-\frac{\lambda}{4}\right)}{\Gamma\left(\frac{1}{4}-\frac{\lambda}{4}\right)},
$$

with the extreme values of $t$ corresponding to $\phi_{ \pm}$. This follows from the asymptotic properties of $\Phi$ for large $x$ [29, Sec. 6.13.1].

Applying (16) with $c=1 / 2$ and $a=1 / 4-\lambda_{0} / 4$, c.f. (20), it follows that algebraic deformations only occur when the factorization energy $\lambda_{0}$ is an odd integer. We rule out $y_{1}(z)$ and $y_{2}(z)$ because we need $\lambda_{0}<1$. We rule out $y_{4}(z)$, because (22) shows that the corresponding eigenfunction always has node. Thus, we are left with factorization functions of the form

$$
\phi_{\mathrm{ho}}^{(m)}(x)=e^{-\frac{x^{2}}{2}} y_{3}\left(x^{2}\right)=\phi_{\mathrm{ho}}(x ;-1-4 m, 1,0) \propto e^{\frac{x^{2}}{2}} H_{2 m}(i x) .
$$


In this way we obtain the following algebraic deformations of the harmonic oscillator:

$$
U_{\mathrm{ho}}^{(m)}(x)=x^{2}-2 \partial_{x x}\left(\log H_{2 m}(i x)\right)-2, \quad m=0,1,2, \ldots
$$

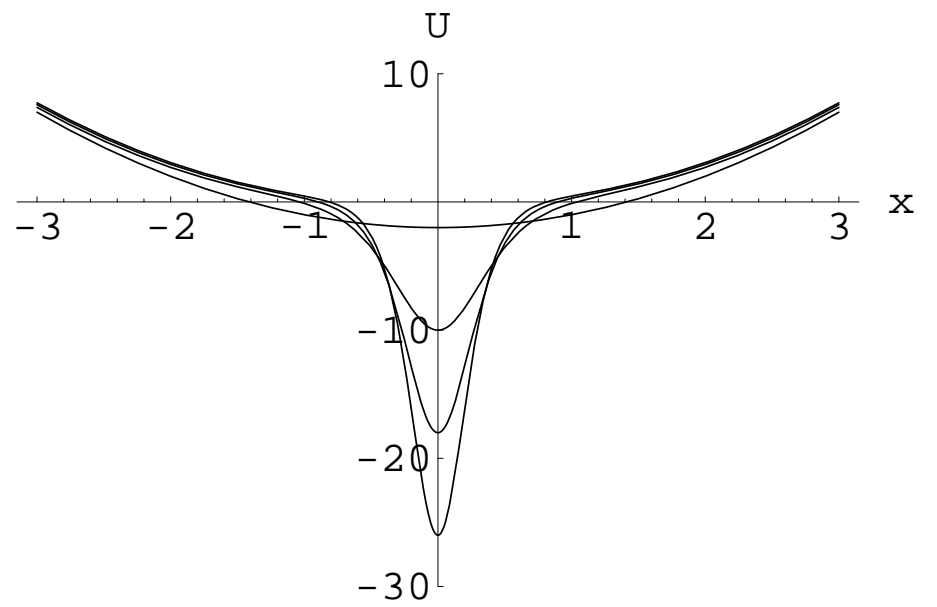

Figure 1. Deformations $U_{\mathrm{ho}}^{(m)}(x)$ of the harmonic oscillator for $m=0,1,2,3$

The harmonic oscillator is shape invariant, and so the zeroth deformation is again a harmonic oscillator, albeit with a spectral shift. The potentials and eigenfunctions of the higher deformations are described in [30,31]. The full, two parameter family of backward transformations is discussed in [9].

The new spectral minimum is $-1-4 m$, and the new ground state is a multiple of

$$
\psi_{\mathrm{ho}, 0}^{(m)}(x) \propto\left(\phi_{\mathrm{ho}}^{(m)}(x)\right)^{-1} \propto e^{-\frac{x^{2}}{2}}\left(H_{2 m}(i x)\right)^{-1} .
$$

The rest of the spectrum is unchanged. The Darboux transformation corresponds to the operator

$$
\begin{aligned}
\alpha_{\mathrm{ho}}^{(m)} & =\partial_{x}-\partial_{x} \log \left(e^{\frac{x^{2}}{2}} H_{2 m}(i x)\right) \\
& =\partial_{x}-x-\frac{4 i m H_{2 m-1}(i x)}{H_{2 m}(i x)} .
\end{aligned}
$$

Consequently, the higher bound states are

$$
\psi_{\mathrm{ho}, j}^{(m)} \propto \alpha_{\mathrm{ho}}^{(m)}\left[\psi_{\mathrm{ho}, j-1}\right]=e^{-\frac{x^{2}}{2}}\left(H_{2 m}(i x)\right)^{-1} p_{j}^{(m)}(x), \quad j=1,2, \ldots,
$$

where

$$
\begin{aligned}
p_{j}^{(m)}(x)= & 2(j-1) H_{2 m}(i x) H_{j-2}(x)-2 x H_{2 m}(i x) H_{j-1}(x) \\
& -4 i m H_{2 m-1}(i x) H_{j-1}(x) .
\end{aligned}
$$

Thus, the even polynomials

$$
q_{k}(z)=p_{2 k}^{(m)}(x), \quad z=x^{2}, \quad k=1, \ldots, n
$$

together with $q_{0}=1$, span an invariant $(n+1)$-dimensional submodule of $\mathcal{P}_{n+m}$. The odd polynomials

$$
r_{k}(z)=x^{-1} p_{2 k+1}^{(m)}(x), \quad k=0, \ldots, n
$$


also span an $(n+1)$-dimensional invariant submodule of $\mathcal{P}_{n+m}$. Therefore, algebraic deformations of the harmonic oscillator are exactly solvable by polynomials.

\subsection{The Morse potential.}

The Morse potential [18] has the form

$$
U_{\text {mo }}(x)=-\left(A+\frac{1}{2}\right) e^{-x}+\frac{1}{4} e^{-2 x} .
$$

The function

$$
\phi_{\mathrm{mo}}\left(x ; k, C_{+}, C_{-}\right)=\sum_{ \pm} C_{ \pm} e^{ \pm k x-\frac{1}{2} e^{-x}} \Phi\left(\mp k-A, 1 \mp 2 k, e^{-x}\right)
$$

is, generically, the general solution of the corresponding Schrödinger equation

$$
-\phi^{\prime \prime}(x)+U_{\text {mo }}(x) \phi(x)=-k^{2} \phi .
$$

In the singular case where $1-2 k$ is a non-positive integer, the general solution can be given as

$$
\begin{aligned}
\phi_{\mathrm{mo}}\left(x ; k, C_{+}, C_{-}\right)= & C_{+} e^{k x-\frac{1}{2} e^{-x}} \Psi\left(-k-A, 1-2 k, e^{-x}\right) \\
& +C_{-} e^{-k x-\frac{1}{2} e^{-x}} \Phi\left(k-A, 1+2 k, e^{-x}\right),
\end{aligned}
$$

where $\Psi$ is the irregular solution of the confluent hypergeometric equation.

There are no bound states if $A \leq 0$, and $\lceil A\rceil$ bound states otherwise, with the $n^{\text {th }}$ bound state is

$$
\psi_{\mathrm{mo}, n}(x) \propto \phi_{\mathrm{mo}}(x, A-n, 0,1) \propto e^{(n-A) x-\frac{1}{2} e^{-x}} L_{n}^{2(A-n)}\left(e^{-x}\right), \quad 0 \leq n<A .
$$

We will focus on deformations of potentials with bound states only. For $A>0$, the spectral minimum is $-A^{2}$, and hence we must have

$$
A<|k| \text {. }
$$

In the non-singular case we have by [29, Sec. 6.13.1]

$$
\begin{aligned}
& \phi_{+}=\phi_{\mathrm{mo}}(x ; k, 0,1), \\
& \phi_{-}=\phi_{\mathrm{mo}}\left(x ; k, 1,-\frac{\Gamma(1-2 k)}{\Gamma(1+2 k)} \frac{\Gamma(k-A)}{\Gamma(-k-A)}\right) .
\end{aligned}
$$

In the singular cases, when $2 k-1$ or $A+k$ is $0,1,2, \ldots$, we have

$$
\phi_{-}=\phi_{\mathrm{mo}}(x ; k, 1,0) \text {. }
$$

The above hold for $k>A$. For $k<-A$, the order of $C_{+}, C_{-}$is reversed.

To obtain algebraic deformations we apply (16) with $a=-k-A$ and $c=1-2 k$, and consider the four possible factorization functions

$$
\phi_{i}(x)=e^{k x-\frac{1}{2} e^{-x}} y_{i}\left(e^{-x}\right), \quad i=1,2,3,4,
$$

in turn. 
Let $m$ be a non-negative integer. For $\phi_{1}$ we need $k+A=m$, which by (28) implies that $A<\frac{m}{2}$. Hence, by (25) (31),

$$
\phi_{1}(x)=\phi_{\mathrm{mo}}(x ; m-A, 1,0)=\phi_{-}(x),
$$

and therefore it generates an isospectral deformation.

For $\phi_{2}(x)$ we need $1-k+A=m$, which by (28) implies $A<\frac{m}{2}-\frac{1}{2}$. Hence, by (25) (29),

$$
\phi_{2}(x)=\phi_{\mathrm{mo}}(x ; 1+A-m, 0,1)=\phi_{+}(x),
$$

and therefore it also generates an isospectral deformation.

For $\phi_{3}(x)$ we need $k-1-A=m$, and hence by (25)

$$
\phi_{3}(x)=\phi_{\mathrm{mo}}(x ; m+1+A, 1,0) .
$$

By (30) this function is nodeless if and only if

$$
\frac{\Gamma(1-2 k)}{\Gamma(-k-A)}=\frac{\Gamma(-1-2 A-2 m)}{\Gamma(-1-2 A-m)}>0,
$$

which holds for even $m$, and fails for odd.

For $\phi_{4}(x)$ we need $k+A=-m$. Hence,

$$
\phi_{4}(x)=\phi_{\mathrm{mo}}(x ;-m-A, 0,1)=\phi_{+}(x)
$$

and therefore it generates an isospectral deformation.

It follows that the only algebraic deformations of the Morse potential corresponding to backward transformations, correspond to the factorization function

$$
\phi_{\text {mo }}^{(m)}(x)=\phi_{3}(x) \propto e^{(m+A+1) x+\frac{1}{2} e^{-x}} L_{m}^{-2(1+m+A)}\left(-e^{-x}\right), \quad A>0, \quad m \text { even. }
$$

The resulting potentials have the form

$$
U_{\mathrm{mo}}^{(m)}(x)=-\left(A+\frac{3}{2}\right) \mathrm{e}^{-x}+\frac{1}{4} \mathrm{e}^{-2 x}-2 \partial_{x x}\left(\log L_{m}^{-2(1+m+A)}\left(-e^{-x}\right)\right) .
$$

The Darboux transformation corresponds to the operator

$$
\begin{aligned}
\alpha_{\mathrm{mo}}^{(m)} & =\partial_{x}-\partial_{x}\left(\log \phi_{\mathrm{mo}}^{(m)}\right) \\
& =\partial_{x}-(1+m+A)+\frac{1}{2} e^{-x}+\frac{e^{-x} r_{m}^{\prime}\left(e^{-x}\right)}{r_{m}\left(e^{-x}\right)},
\end{aligned}
$$

where

$$
r_{m}(z)=L_{m}^{-2(1+m+A)}(-z) .
$$

Applying $\alpha_{\text {mo }}^{(m)}$ to the bound states (27), we infer that the bound states of the deformed potential are

$$
\psi_{\mathrm{mo}, j}^{(m)}(x)=\left(\frac{e^{(j-A) x-\frac{1}{2} e^{-x}}}{r_{m}\left(e^{-x}\right)}\right) p_{j}^{(m)}\left(e^{-x}\right),
$$

where

$$
\begin{aligned}
& p_{j}^{(m)}(z)=q_{j}(z) r_{m}(z)(z+n-m-2 A-1)-z q_{j}^{\prime}(z) r_{m}(z)+z q_{j}(z) r_{m}^{\prime}(z) \\
& q_{j}(z)=L_{j}^{2(A-j)}(z) .
\end{aligned}
$$


Note that the $p_{j}^{(m)}(z)$ are polynomials of degree $j+m+1$, and hence, for every $n$, the polynomials

$$
1, p_{0}^{(m)}(z), p_{1}^{(m)}(z), \ldots, p_{n}^{(m)}(z)
$$

span a codimension $m$ invariant subspace of $\mathcal{P}_{m+n+1}(z)$. We have demonstrated that algebraic deformations of the Morse potential are exactly solvable by polynomials.

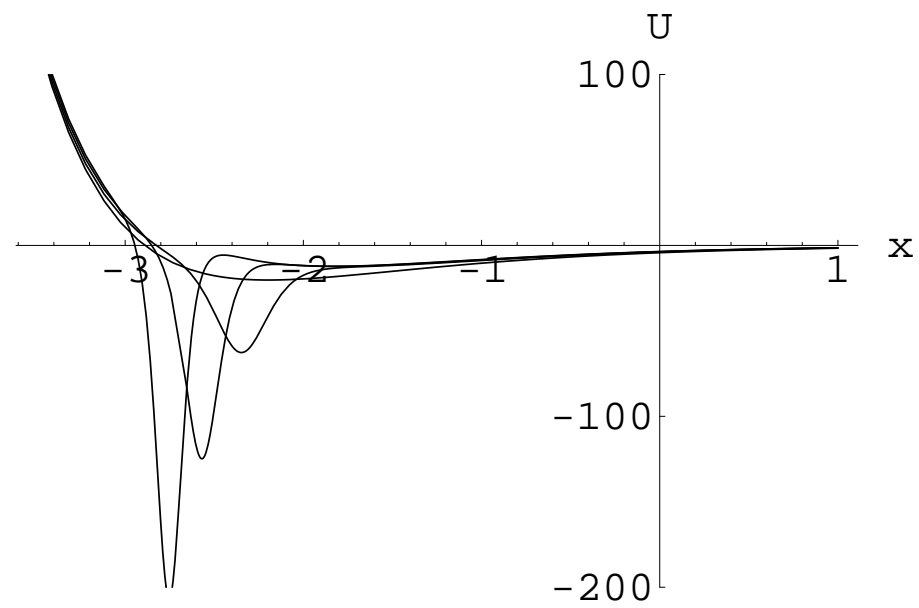

Figure 2. Algebraic deformations $U^{(m)}(x)$ of the Morse potential for $A=2.5$ and $m=0,1,2$ and 3 .

\subsection{The hyperbolic Pöschl-Teller potential.}

The hyperbolic Pöschl-Teller potential [19, which includes the class of reflectionless 1-soliton potentials 32 , has the form

$$
U_{\mathrm{pt}}(x)=\frac{1}{4}\left(\frac{1}{4}-A^{2}\right) \operatorname{sech}^{2}\left(\frac{x}{2}\right) .
$$

The general solution [29, Sec. 2.9] of the corresponding Schrödinger equation

$$
-\phi^{\prime \prime}(x)+U_{\mathrm{pt}}(x) \phi(x)=-k^{2} \phi(x)
$$

can be given as

$$
\begin{aligned}
\phi_{\mathrm{pt}}\left(x ; k, C_{0}, C_{1}\right)= & \cosh \left(\frac{x}{2}\right)^{\frac{1}{2}-A}\left\{C_{0} F\left(-\frac{A}{2}+\frac{1}{4}+k,-\frac{A}{2}+\frac{1}{4}-k, \frac{1}{2} ;-\sinh ^{2}\left(\frac{x}{2}\right)\right)\right. \\
& \left.+C_{1} \sinh \left(\frac{x}{2}\right) F\left(-\frac{A}{2}+\frac{3}{4}+k,-\frac{A}{2}+\frac{3}{4}-k, \frac{3}{2} ;-\sinh ^{2}\left(\frac{x}{2}\right)\right)\right\},
\end{aligned}
$$

where $F(a, b, c ; z)$ also denotes the analytic continuation of the hypergeometric function to $\operatorname{Re}(z)<0$. For $A>1 / 2$, the potential (34) has $\left\lceil A-\frac{1}{2}\right\rceil$ bound states

$$
\psi_{\mathrm{pt}, j}(x), \quad 0 \leq j<A-\frac{1}{2} .
$$

The even bound states are

$$
\begin{aligned}
\psi_{\mathrm{pt}, 2 i}(x) & \propto \phi_{\mathrm{pt}}\left(x ; \frac{A}{2}-i-\frac{1}{4}, 1,0\right) \\
& \propto \cosh \left(\frac{x}{2}\right)^{\frac{1}{2}-A} P_{i}^{\left(-\frac{1}{2},-A\right)}(\cosh x) .
\end{aligned}
$$


The odd ones are

$$
\begin{aligned}
\psi_{\mathrm{pt}, 2 i+1}(x) & \propto \phi_{\mathrm{pt}}\left(x ; \frac{A}{2}-i-\frac{3}{4}, 0,1\right) \\
& \propto \sinh \left(\frac{x}{2}\right) \cosh \left(\frac{x}{2}\right)^{\frac{1}{2}-A} P_{i}^{\left(\frac{1}{2},-A\right)}(\cosh x) .
\end{aligned}
$$

We focus on deformations of potentials with bound states only, i.e., $A>\frac{1}{2}$. The spectral minimum is $-\left(\frac{1}{2}-A\right)^{2}$. For $|k|>\frac{1}{2}-A$, the nodeless solutions of (19) are given by

$$
\phi_{\mathrm{pt}}(x ; k, 1, t), \quad|t| \leq 2 \frac{\Gamma\left(\frac{3}{4}+k-\frac{A}{2}\right) \Gamma\left(\frac{3}{4}+k+\frac{A}{2}\right)}{\Gamma\left(\frac{1}{4}+k-\frac{A}{2}\right) \Gamma\left(\frac{1}{4}+k+\frac{A}{2}\right)},
$$

with the extreme values of $t$ corresponding to $\phi_{ \pm}$[29, Sec. 2.3.2, Sec. 2.10].

To obtain algebraic deformations we apply (18) with

$$
a=\frac{1}{4}+k-\frac{A}{2}, \quad b=\frac{1}{4}-k-\frac{A}{2}, \quad c=1-A,
$$

and consider the four possible factorization functions

$$
\phi_{i}(x)=\cosh \left(\frac{x}{2}\right)^{\frac{1}{2}-A} f_{i}\left(\cosh ^{2}\left(\frac{x}{2}\right)\right), \quad i=1,2,3,4 .
$$

We rule out $\phi_{2}(x)$ and $\phi_{3}(x)$ because these are odd functions, and hence have a node. The factorization functions of the form

$$
\begin{aligned}
\phi_{1}(x) & =\phi_{\mathrm{pt}}\left(A, \frac{A}{2}-\frac{1}{4}-m, 1,0\right) \\
& \propto \cosh \left(\frac{x}{2}\right)^{\frac{1}{2}-A} P_{m}^{\left(-\frac{1}{2},-A\right)}(\cosh (x))
\end{aligned}
$$

are nodeless for $m>A-\frac{1}{2}$. The factorization functions of the form

$$
\begin{aligned}
\phi_{4}(x) & =\phi_{\mathrm{pt}}\left(A,-\frac{A}{2}-\frac{1}{4}-m, 1,0\right) \\
& \propto \cosh \left(\frac{x}{2}\right)^{\frac{1}{2}+A} P_{m}^{\left(-\frac{1}{2}, A\right)}(\cosh (x))
\end{aligned}
$$

are nodeless for all $m=0,1,2, \ldots$.

Thus, we see that there are two series of algebraic deformations. In order to study deformations for all possible $m$, we focus on the latter series. The resulting potentials have the form

$$
U_{\mathrm{pt}}^{(m)}(x)=-\frac{1}{4}\left(A+\frac{1}{2}\right)\left(A+\frac{3}{2}\right) \operatorname{sech}^{2}\left(\frac{x}{2}\right)-2 \partial_{x x}\left(\log P_{m}^{\left(-\frac{1}{2}, A\right)}(\cosh x)\right) .
$$

The Darboux transformation corresponds to the operator

$$
\alpha_{\mathrm{pt}}^{(m)}=\partial_{x}-\partial_{x} \log \phi_{4}(x)
$$

Applying $\alpha_{\mathrm{pt}}^{(m)}$ to the even bound state functions (35) yields

$$
\psi_{\mathrm{pt}, 2 j}^{(m)}(x)=\mu^{(m)}(x) s_{j}^{(m)}(w)
$$

where

$$
\begin{aligned}
\mu^{(m)}(x) & =\frac{\cosh \left(\frac{x}{2}\right) \sinh (x)}{(w+1) q_{m}(w)} \\
w & =2 z+1=\cosh (x) \\
s_{j}^{(m)}(w) & =(w+1)\left\{p_{j}^{\prime}(w) q_{m}(w)-q_{m}^{\prime}(w) p_{j}(w)\right\}-A q_{m}(w) p_{j}(w) \\
q_{m}(w) & =P_{m}^{\left(-\frac{1}{2}, A\right)}(w) \\
p_{j}(w) & =P_{j}^{\left(-\frac{1}{2},-A\right)}(w) .
\end{aligned}
$$




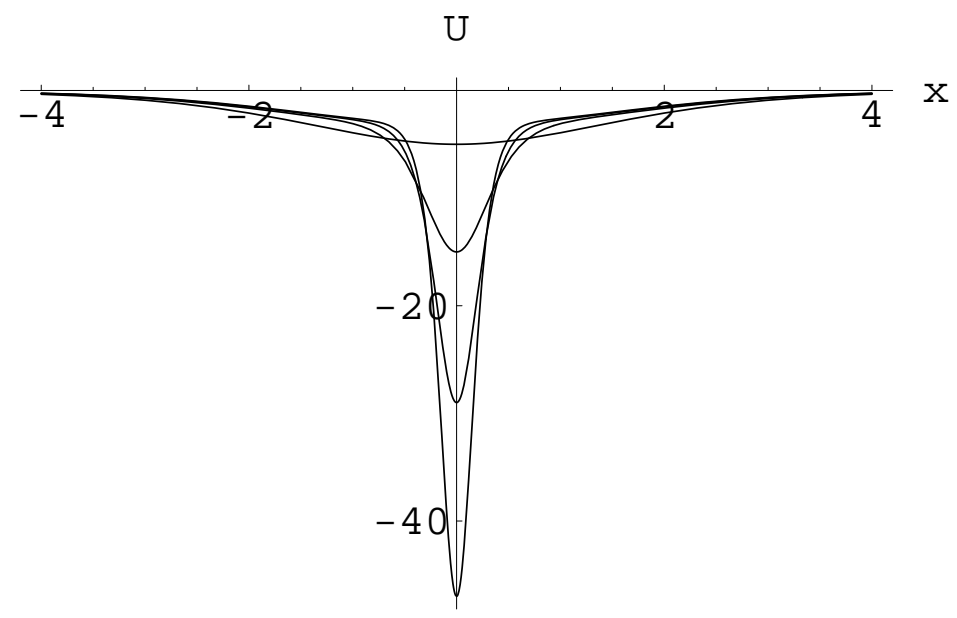

Figure 3. Algebraic deformations $U_{\mathrm{pt}}^{(m)}(x)$ of the hyperbolic Pöschl-Teller potential (39), with $A=4$ and $m=0,1,2$ and 3 .

Hence, the deformed operator, conjugated by the gauge factor $\mu^{(m)}(x)$, preserves the codimension $m$ submodule of $\mathcal{P}_{m+n}(z)$ spanned by $s_{j}^{(m)}(w), j=0, \ldots, n$. A similar result holds for the deformation of the odd bound states (36). Therefore, algebraic deformations of the hyperbolic Pöschl-Teller potential are exactly solvable by polynomials.

\section{Exceptional monomial modules.}

In this section we characterize the algebraic structure of the first-fold deformations $(m=1)$ described in the preceding section. We will show that this is precisely the class of exactly solvable operators that preserves the infinite flag of polynomial modules

$$
\mathcal{P}_{0}^{(1)} \subset \mathcal{P}_{2}^{(1)} \subset \mathcal{P}_{3}^{(1)} \subset \ldots \subset \mathcal{P}_{n}^{(1)} \subset \ldots,
$$

where

$$
\mathcal{P}_{n}^{(1)}=\operatorname{span}\left\{1, z^{2}, z^{3}, \ldots, z^{n}\right\}, \quad \mathcal{P}_{0}^{(1)}=\operatorname{span}\{1\} .
$$

Will will call such modules exceptional monomial modules. They are exceptional in the sense that the family of second order operators that leave them invariant is very rich [22,25]. To this effect, let us begin by the following

Proposition 2 A second-order differential operator preserves $\mathcal{P}_{n}^{(1)}$ if and only if it is a linear combination of the following 7 operators:

$$
\begin{aligned}
& T_{2}^{(+2)}=z^{4} \partial_{z z}+2(1-n) z^{3} \partial_{z}+n(n-1) z^{2} \\
& T_{2}^{(+1)}=z^{3} \partial_{z z}-(n-1) z^{2} \partial_{z}, \\
& T_{2}^{(0)}=z^{2} \partial_{z z} \\
& T_{2}^{(-1)}=z \partial_{z z}-\partial_{z} \\
& T_{2}^{(-2)}=\partial_{z z}-2 z^{-1} \partial_{z},
\end{aligned}
$$




$$
\begin{aligned}
& T_{1}^{(0)}=z \partial_{z}, \\
& T_{0}^{(0)}=1 .
\end{aligned}
$$

A proof of the Propostion is found in [22,25]. If the linear combination contains the raising operators $T_{2}^{(+2)}$ and $T_{2}^{(+1)}$ then the operator will preserve $P_{n}^{(1)}$ but not the whole flag (41). These cases are called quasi-exactly solvable in the literature 15, 16, 17] and will be analyzed in detail in 25]. Since we restrict to exactly solvable cases, we shall consider only the following linear combination

$$
T=p_{2} T_{2}^{(0)}+p_{1} T_{2}^{(-1)}+p_{0} T_{2}^{(-2)}+q_{2} T_{1}^{(0)},
$$

where the additive constant has been neglected. This can be written as

$$
T=P(z) \partial_{z z}+Q(z) \partial_{z},
$$

where

$$
\begin{aligned}
& P(z)=p_{2} z^{2}+p_{1} z+p_{0}, \\
& Q(z)=q_{2} z-p_{1}-2 p_{0} z^{-1},
\end{aligned}
$$

are quadratic polynomials whose coefficients $p_{2}, p_{1}, p_{0}$ and $q_{2}$ are arbitrary real numbers.

The exceptional monomial module $\mathcal{P}_{n}^{(1)}$ is invariant with respect to scaling of the $z$ variable. By also allowing rescaling of the physical variable $x$, it suffices to consider the following canonical cases.

\begin{tabular}{c|ccc} 
& $P(z)$ & $Q(z)$ & $z(x)$ \\
\hline Ia & $(1-z)(z+2+2 A)$ & $q_{2} z+2 A+1-4(1+A) z^{-1}$ & $\left(\frac{3}{2}+A\right) \cosh ^{2}(x)-A-\frac{1}{2}$ \\
Ib & $z(1-z)$ & $q_{2} z-1$ & $\cosh ^{2}\left(\frac{x}{2}\right)$ \\
Ic & $-\left(1+z^{2}\right)$ & $q_{2} z+2 z^{-1}$ & $\sinh x$ \\
IIa & $-(z-1)^{2}$ & $q_{2} z-2+2 z^{-1}$ & $-(2 A+3) e^{x}+1$ \\
IIb & $-z^{2}$ & $q_{2} z$ & $e^{x}$ \\
IIIa & $8(1-z)$ & $q_{2} z+8-16 z^{-1}$ & $2 x^{2}+1$ \\
IIIb & $-4 z$ & $q_{2} z+1$ & $x^{2}$ \\
IV & -1 & $q_{2} z+2 z^{-1}$ & $x$
\end{tabular}

Table 2. Second-order operators preserving the exceptional monomial module

In cases Ib, IIb, IIIb, the operator is of the form shown in (13), and therefore preserves the full $\mathcal{P}_{n}$ and not just the exceptional module $\mathcal{P}_{n}^{(1)}$. Thus, these cases describe undeformed shape-invariant potentials. Cases Ic and IV correspond to singular potentials, and will not be discussed further.

Proposition 1 shows that the non-singular potentials in case Ia correspond to

$$
q_{2}= \pm\left(A+\frac{3}{2}\right)
$$

Both possibilities yield the same potential form, so we take the former. Using (111) (391) we have

$$
U(x)=-\frac{1}{4}\left(A+\frac{1}{2}\right)\left(A+\frac{3}{2}\right)+2 \frac{k \cosh x-1}{(\cosh x-k)^{2}}, \quad k=\frac{2 A+1}{2 A+3},
$$




$$
=U_{\mathrm{pt}}^{(1)}(x)+\left(\frac{5}{4}+\frac{A}{2}\right)^{2} .
$$

For similar reasons, for case IIIa potentials we must take $q_{2}=4$. Using (11) (23) we obtain

$$
\begin{aligned}
U(x) & =3+x^{2}+\frac{8}{2 x^{2}+1}-\frac{16}{\left(2 x^{2}+1\right)^{2}}, \\
& =U_{\mathrm{ho}}^{(1)}(x)+5
\end{aligned}
$$

For the case IIa, we translate the $x$ variable and do a spectral shift to set $q_{2}=2 A+3$. In this way, (11) (33) yields

$$
\begin{aligned}
U(x) & =(2+A)^{2}+\frac{1}{4} e^{-2 x}-\left(A+\frac{3}{2}\right) e^{-x}+\frac{2 k e^{x}}{\left(1-k e^{x}\right)^{2}}, \quad k=2 A+3, \\
& =U_{\text {mo }}^{(1)}(x)+(2+A)^{2} .
\end{aligned}
$$

We should note that the above potential form is non-singular only if $2 A+3<0$. This is unavoidable, in as much as we showed in section 3.5 that the odd deformation of Morse potentials with bound states produce singular potentials.

In summary, we have demonstrated that non-singular Hamiltonians that are exactly solvable by an infinite flag of exceptional monomial modules and not by the ordinary flag (II) are precisely the first-fold algebraic deformations of the non-singular shape-invariant potentials.

Although these new potentials preserve a full flag of polynomial subspaces, and therefore are exactly solvable in the sense defined by Turbiner in [15, they do not possess a hidden $\mathfrak{s l}(2)$ symmetry algebra structure. This shows that the exactly solvable class is wider than the Lie-algebraic one.

\section{Acknowledgments}

The research of DGU is supported in part by a CRM-ISM Postdoctoral Fellowship and the Spanish Ministry of Education under grant EX2002-0176. The research of NK and RM is supported by the National Science and Engineering Research Council of Canada. The authors would like to thank Prof. González-López and Prof. Gesztesy for interesting discussions, as well as the referees, who made very interesting remarks on the first version of the paper.

[1] Darboux G, Théorie Générale des Surfaces, vol. II, Gauthier-Villars, 1888.

[2] Jacobi CG, 1837 J. Reine Angew. Math. 17, 68.

[3] Schrödinger E 1941 Proc. Roy. Irish Acad. 47 A, 53 (Preprint physics/9910003).

[4] Infeld L and Hull T E 1951 Rev. Mod. Phys. 2321.

[5] Cooper F, Khare A and Sukhatme U 1995 Phys. Rep. 251267.

[6] Deift P and Trubowitz E 1979 Duke Math J. 45, 267.

[7] Gesztesy F, Simon B and Teschl G 1996 J. d'Analyse Math. 70, 267

[8] Calogero F and Degasperis A 1982 Spectral transform and solitons I, Studies in Mathematics and its Applications (New York:Elsevier).

[9] Sukumar CV 1985 J. Phys. A 182917. 
[10] Sparenberg J-M and Baye D 1995 J. Phys. A 285079.

[11] Bagrov V G and Samsonov B F 1995 Theoret. and Math. Phys. 1041051.

[12] Gendenshtein L 1983 JETP Lett 38356.

[13] Mielnik B 1984 J. Math. Phys. 253387.

[14] Lévai G, Baye D and Sparenberg J-M 1997 J. Phys. A 308257

[15] Turbiner A V 1988 Commun. Math. Phys. 118467.

[16] Kamran N and Olver P J 1990 J. Math. Anal. Appl. 145342.

[17] González-Lopez A, Kamran N and Olver P J 1993 Commun. Math. Phys. 153117.

[18] Morse P M 1929 Phys. Rev. 5757.

[19] Pöschl G and Teller E 1933 Z. Physik 83143.

[20] Milson R 1998 Internat. J. Theoret. Phys. 371735.

[21] Gómez-Ullate D, González-López A and Rodríguez M A 2000 J. Phys. A 337305.

[22] Post G and Turbiner A V 1995 Russian J. Math. Phys. 3113.

[23] Finkel F and Kamran N 1998 Adv. in Applied Math. 20300.

[24] Baye D, Sparenberg J-M and Lévai G 1997 Inverse and Algebraic Quantum Scattering Theory (Lecture notes in Physics 488) ed B Apagyi, G Endrédi and P Lévay (Berlin: Springer) p 295

[25] Gómez-Ullate D, Kamran N and Milson R, in preparation.

[26] González-López A and Tanaka T, hep-th/0307094

[27] Shifman M 1989 Int. J. Modern Phys. A 43311.

[28] Schminke U W 1978, Proc. Roy. Soc. Edinburgh Sec. A 80, 67.

[29] Erdélyi A et al. 1953 Higher Transcendental Functions, Vol. I, (New York:McGraw-Hill).

[30] Dubov S Y, Eleonskii V M and Kulagin N E 1992, Sov. Phys. JETP 75446.

[31] Bagrov V G and Samsonov B F 1997 Pramana J. Phys. 49563.

[32] Matveev V and Salle M A 1991 Darboux transformations and solitons, Springer Series in Nonlinear Dynamics (Berlin:Springer) 